\title{
Topotactic oxidative and reductive control of the structures and properties of layered manganese oxychalcogenides.
}

\author{
Geoffrey Hyett, Nicolas Barrier, and Simon J. Clarke*
}

Department of Chemistry, University of Oxford, Inorganic Chemistry Laboratory, South Parks Road, Oxford, OX1 3QR, UK.

\section{Joke Hadermann}

Electron Microscopy for Materials Science (EMAT), University of Antwerp,

Groenenborgerlaan 171, B-2020 Antwerp, Belgium.

\section{Supporting Information}

E-mail: $\quad$ simon.clarke@ chem.ox.ac.uk

Fax: $\quad$ +44 1865272690

Tel: $\quad$ +44 1865272600 
Table S1. Refined structural parameters for an as-made Mn-rich oxysulfide derived from SXRD data. $U_{\text {eq }}$ measured in $\AA^{2}$. See Table 1 in main text for refinement results.

\begin{tabular}{|c|c|c|}
\hline & Compound & $\mathrm{Sr}_{4} \mathrm{Mn}_{2.94} \mathrm{O}_{7.42} \mathrm{Cu}_{2} \mathrm{~S}_{2}$ \\
\hline & Sample ID & NB17 \\
\hline Atom & Parameter & \\
\hline \multirow[t]{2}{*}{$\operatorname{Sr} 1(1 / 21 / 2 z)$} & $z$ & $0.05980(2)$ \\
\hline & $100 \times U_{\mathrm{eq}}$ & $2.50(2)$ \\
\hline \multirow[t]{2}{*}{$\operatorname{Sr} 2(1 / 21 / 2 z)$} & $z$ & $0.166407(16)$ \\
\hline & $100 \times U_{\mathrm{eq}}$ & $0.99(1)$ \\
\hline \multirow[t]{2}{*}{ Mn1 $\left(\begin{array}{llll}0 & 0 & z\end{array}\right)$} & $z$ & $0.11381(3)$ \\
\hline & $100 \times U_{\text {eq }}$ & $0.97(2)$ \\
\hline \multirow[t]{2}{*}{$\operatorname{Mn} 2(0,0,0)$} & $100 \times U_{\text {eq }}$ & $1.22(4)$ \\
\hline & Occupancy & $0.936(6)$ \\
\hline $\mathrm{Cu}(1 / 201 / 4)$ & $100 \times U_{\text {eq }}$ & $1.95(2)$ \\
\hline \multirow[t]{2}{*}{$\mathrm{S}\left(\begin{array}{lll}0 & 0 & z\end{array}\right)$} & $\mathrm{z}$ & $0.20828(4)$ \\
\hline & $100 \times U_{\mathrm{eq}}$ & $1.12(3)$ \\
\hline \multirow[t]{2}{*}{ O1 $\left(\begin{array}{llll}0 & 0 & z\end{array}\right)$} & $\mathrm{Z}$ & $0.05432(16)$ \\
\hline & $100 \times U_{\mathrm{eq}}$ & $2.97(14)$ \\
\hline \multirow[t]{3}{*}{$\mathrm{O} 2(1 / 2$ y 0$)$} & $y$ & $0.070(8)$ \\
\hline & $100 \times U_{\text {eq }}$ & $4.4(9)$ \\
\hline & Occupancy & $0.356(12)$ \\
\hline \multirow[t]{2}{*}{$\mathrm{O} 3\left(\begin{array}{lll}(1 / 2 & 0 & z\end{array}\right)$} & $z$ & $0.11957(9)$ \\
\hline & $100 \times U_{\text {eq }}$ & $1.41(5)$ \\
\hline
\end{tabular}

${ }^{a} U_{\text {eq }}$ is defined as one third of the orthogonalized $U_{i j}$ tensor. 
Table S2. Selected bond distances and angles for an as-made Mn-rich oxysulfide derived from SXRD data.

\begin{tabular}{|l|l|}
\hline Compound & $\mathrm{Sr}_{4} \mathrm{Mn}_{2.94} \mathrm{O}_{7.42} \mathrm{Cu}_{2} \mathrm{~S}_{2}$ \\
\hline Sample ID & $\mathrm{NB} 17$ \\
\hline Bond distance / $\AA$ & $2.051(5)$ \\
\hline $\mathrm{Mn} 1-\mathrm{O} 1[1]$ & $1.9624(3)$ \\
\hline $\mathrm{Mn} 1-\mathrm{O} 3$ [4] & $1.872(5)$ \\
\hline $\mathrm{Mn} 2-\mathrm{O} 1[2]$ & $-{ }^{a}$ \\
\hline $\mathrm{Mn} 2-\mathrm{O} 2[4]$ & $2.4248(9)$ \\
\hline $\mathrm{Cu}-\mathrm{S}[4]$ & \\
\hline $\mathrm{Bond}$ angle / & \\
\hline $\mathrm{O} 1-\mathrm{Mn} 1-\mathrm{O} 3[4]$ & $95.80(9)$ \\
\hline $\mathrm{O} 3-\mathrm{Mn} 1-\mathrm{O} 3[4]$ & $89.41(2)$ \\
\hline $\mathrm{S}-\mathrm{Cu}-\mathrm{S}[2]$ & $107.24(6)$ \\
\hline $\mathrm{S}-\mathrm{Cu}-\mathrm{S}[4]$ & $110.60(3)$ \\
\hline
\end{tabular}

${ }^{a}$ omitted due to structural disorder in $\mathrm{O} 2$ position 
Table S3. Results of PND refinements at $295 \mathrm{~K}$ for Mn-rich oxysulfides derived from the $\mathrm{Sr}_{4} \mathrm{Mn}_{2.91} \mathrm{O}_{7.40} \mathrm{Cu}_{2} \mathrm{~S}_{2}$ parent from PND data collected on POLARIS.

\begin{tabular}{|c|c|c|c|c|}
\hline Compound & $\mathrm{Sr}_{4} \mathrm{Mn}_{2.91} \mathrm{O}_{6.97} \mathrm{Cu}_{2} \mathrm{~S}_{2}$ & $\mathrm{Sr}_{4} \mathrm{Mn}_{2.91} \mathrm{O}_{7.05} \mathrm{Cu}_{2} \mathrm{~S}_{2}$ & $\mathrm{Sr}_{4} \mathrm{Mn}_{2.91} \mathrm{O}_{7.40} \mathrm{Cu}_{2} \mathrm{~S}_{2}$ & $\mathrm{Sr}_{4} \mathrm{Mn}_{2.91} \mathrm{O}_{7.40} \mathrm{~F}_{0.06} \mathrm{Cu}_{2} \mathrm{~S}_{2}$ \\
\hline Sample ID & GH206 & GH195 & GH192 & GH197 \\
\hline Radiation & \multicolumn{4}{|c|}{ Neutron } \\
\hline Instrument & \multicolumn{4}{|c|}{ POLARIS } \\
\hline Physical form & Black powder & Black powder & Black powder & Black powder \\
\hline$T / \mathrm{K}$ & 295 & 295 & 295 & 295 \\
\hline Space group & $I 4 / \mathrm{mmm}$ & $I 4 / \mathrm{mmm}$ & $\mathrm{I} / \mathrm{mmm}$ & $I 4 / \mathrm{mmm}$ \\
\hline$a / \AA$ & $3.9080(7)$ & $3.89636(4)$ & $3.87248(3)$ & $3.86798(4)$ \\
\hline$c / \AA$ & $34.697(6)$ & $34.5920(5)$ & $34.5892(4)$ & $34.6537(4)$ \\
\hline$V / \AA^{3}$ & $529.9(3)$ & $525.16(2)$ & $518.70(1)$ & $518.46(1)$ \\
\hline $\mathrm{Z}$ & 2 & 2 & 2 & 2 \\
\hline$R_{w p}$ & 0.0168 & 0.0143 & 0.0196 & 0.0210 \\
\hline$R_{F 2}$ & 0.081 & 0.055 & 0.044 & 0.078 \\
\hline$\chi^{2}$ & 1.398 & 2.000 & 2.290 & 2.615 \\
\hline
\end{tabular}


Table S4. Refined structural parameters for Mn-rich oxysulfides derived from the $\mathrm{Sr}_{4} \mathrm{Mn}_{2.91} \mathrm{O}_{7.40} \mathrm{Cu}_{2} \mathrm{~S}_{2}$ parent from PND data collected on POLARIS. $U_{\text {iso }}, U_{\text {eq }}$ measured in $\AA^{2}$

\begin{tabular}{|c|c|c|c|c|c|}
\hline & Compound & $\mathrm{Sr}_{4} \mathrm{Mn}_{2.91} \mathrm{O}_{6.97} \mathrm{Cu}_{2} \mathrm{~S}_{2}$ & $\mathrm{Sr}_{4} \mathrm{Mn}_{2.91} \mathrm{O}_{7.05} \mathrm{Cu}_{2} \mathrm{~S}_{2}$ & $\mathrm{Sr}_{4} \mathrm{Mn}_{2.91} \mathrm{O}_{7.40} \mathrm{Cu}_{2} \mathrm{~S}_{2}$ & $\mathrm{Sr}_{4} \mathrm{Mn}_{2.91} \mathrm{O}_{7.40} \mathrm{~F}_{0.06} \mathrm{Cu}_{2} \mathrm{~S}_{2}$ \\
\hline & Sample ID & GH206 & GH195 & GH192 & GH197 \\
\hline Atom & Parameter & & & & \\
\hline \multirow[t]{2}{*}{$\operatorname{Sr} 1(1 / 21 / 2 z)$} & $z$ & $0.06293(3)$ & $0.06137(2)$ & $0.05921(2)$ & $0.05823(2)$ \\
\hline & $100 \times U_{\text {eq }}$ & $1.59(4)$ & $1.71(3)$ & $1.32(3)$ & $1.42(3)$ \\
\hline \multirow[t]{2}{*}{$\operatorname{Sr} 2(1 / 21 / 2 z)$} & $z$ & $0.16642(2)$ & $0.16627(2)$ & $0.16677(1)$ & $0.16648(2)$ \\
\hline & $100 \times U_{\text {eq }}$ & $0.57(3)$ & $0.64(2)$ & $0.56(2)$ & $0.63(2)$ \\
\hline \multirow[t]{2}{*}{ Mn1 $\left(\begin{array}{lll}0 & 0 & z\end{array}\right)$} & $z$ & $0.11316(4)$ & $0.11270(3)$ & $0.11279(2)$ & $0.11254(3)$ \\
\hline & $100 \times U_{\text {iso }}$ & $0.24(1)$ & $0.23(1)$ & $0.25(1)$ & $0.36(1)$ \\
\hline \multirow[t]{3}{*}{$\operatorname{Mn} 2(x, x, 0)$} & $x$ & $0.0710(7)$ & $0.0518(6)$ & $0^{b}$ & $0^{b}$ \\
\hline & $100 \times U_{\text {iso }}$ & $1.5(1)$ & $1.13(7)$ & $1.08(4)$ & $0.77(3)$ \\
\hline & Occupancy & $0.228^{b}$ & $0.228^{b}$ & $0.913(6)$ & $0.913^{b}$ \\
\hline $\mathrm{Cu}(1 / 201 / 4)$ & $100 \times U_{\text {eq }}$ & $1.67(3)$ & $1.58(2)$ & $1.60(2)$ & $1.60(3)$ \\
\hline \multirow[t]{2}{*}{$\mathrm{S}\left(\begin{array}{lll}0 & 0 & z\end{array}\right)$} & $\mathrm{z}$ & $0.20869(5)$ & $0.20828(4)$ & $0.20791(4)$ & $0.20773(5)$ \\
\hline & $100 \times U_{\text {iso }}$ & $0.73(3)$ & $0.79(3)$ & $0.92(3)$ & $1.02(3)$ \\
\hline
\end{tabular}




\begin{tabular}{|c|c|c|c|c|c|}
\hline $\mathrm{O} 1\left(\begin{array}{lll}0 & 0 & z\end{array}\right)$ & $\mathrm{z}$ & $0.05359(3)$ & $0.05368(3)$ & $0.05443(2)$ & $0.05466(3)$ \\
\hline & $100 \times U_{\text {eq }}$ & $2.37(5)$ & $2.52(4)$ & $1.97(3)$ & $1.99(3)$ \\
\hline $\mathrm{O} 2\left(\begin{array}{lll}1 \\
1 / 2 & 0 & 0\end{array}\right)$ & $100 \times U_{\mathrm{eq}}$ & $26(2)$ & $13.5(5)$ & $5.8(1)$ & $4.5(1)$ \\
\hline & Occupancy & $0.485(6)$ & $0.525(5)$ & $0.698(4)$ & $0.727(4)^{C}$ \\
\hline $\mathrm{O} 3\left(\begin{array}{lll}1 & 0 & z\end{array}\right)$ & $z$ & $0.11961(2)$ & $0.11938(1)$ & $0.11914(1)$ & $0.11920(1)$ \\
\hline & $100 \times U_{\mathrm{eq}}$ & $1.02(3)$ & $1.04(2)$ & $0.79(2)$ & $0.87(2)$ \\
\hline
\end{tabular}

${ }^{a} U_{\text {eq }}$ is defined as one third of the orthogonalized $U_{i j}$ tensor.

${ }^{b}$ Not refined

${ }^{c}$ Total of occupancy by $\mathrm{O}$ and $\mathrm{F}$ (given the similarity in $\mathrm{O}$ and $\mathrm{F}$ scattering lengths) 
Table S5. Selected bond distances and angles for Mn-rich oxysulfides from PND data collected on POLARIS.

\begin{tabular}{|c|c|c|c|c|}
\hline Compound & $\mathrm{Sr}_{4} \mathrm{Mn}_{2.91} \mathrm{O}_{6.97} \mathrm{Cu}_{2} \mathrm{~S}_{2}$ & $\mathrm{Sr}_{4} \mathrm{Mn}_{2.91} \mathrm{O}_{7.05} \mathrm{Cu}_{2} \mathrm{~S}_{2}$ & $\mathrm{Sr}_{4} \mathrm{Mn}_{2.91} \mathrm{O}_{7.40} \mathrm{Cu}_{2} \mathrm{~S}_{2}$ & $\mathrm{Sr}_{4} \mathrm{Mn}_{2.91} \mathrm{O}_{7.40} \mathrm{~F}_{0.06} \mathrm{Cu}_{2} \mathrm{~S}_{2}$ \\
\hline Sample ID & GH206 & GH195 & GH192 & GH197 \\
\hline \multicolumn{5}{|l|}{ Bond distance / $\AA$} \\
\hline Mn1-O1[1] & $2.067(2)$ & $2.042(1)$ & $2.019(1)$ & $2.006(1)$ \\
\hline Mn1-O3 [4] & $1.9667(4)$ & $1.9618(1)$ & $1.9486(1)$ & $1.9477(1)$ \\
\hline $\mathrm{Mn} 2-\mathrm{O} 1[2]$ & $-^{a}$ & $-^{a}$ & $1.8825(8)$ & $1.894(1)$ \\
\hline $\mathrm{Mn} 2-\mathrm{O} 2[4]$ & $-{ }^{a}$ & $-^{a}$ & $1.93624(2)$ & $1.93399(2)$ \\
\hline $\mathrm{Cu}-\mathrm{S}[4]$ & $2.423(1)$ & $2.4245(9)$ & $2.4225(8)$ & $2.426(1)$ \\
\hline \multicolumn{5}{|l|}{ Bond angle $/{ }^{\circ}$} \\
\hline O1-Mn1-O3 [4] & $96.53(4)$ & $96.76(3)$ & $96.47(3)$ & $96.81(3)$ \\
\hline O3-Mn1-O3 [4] & $89.259(9)$ & $89.205(7)$ & $89.273(6)$ & $89.195(7)$ \\
\hline $\mathrm{S}-\mathrm{Cu}-\mathrm{S}[2]$ & $107.48(7)$ & $106.94(6)$ & $106.12(5)$ & $105.72(6)$ \\
\hline $\mathrm{S}-\mathrm{Cu}-\mathrm{S}[4]$ & $110.48(4)$ & $110.75(3)$ & $111.17(3)$ & $111.38(3)$ \\
\hline
\end{tabular}

\footnotetext{
${ }^{a}$ omitted due to structural disorder in Mn2 position
} 
Table S6. Results of PND refinements at $295 \mathrm{~K}$ for Mn-poor oxysulfides derived from the $\mathrm{Sr}_{4} \mathrm{Mn}_{2.77} \mathrm{O}_{7.27} \mathrm{Cu}_{2} \mathrm{~S}_{2}$ parent from PND data collected on POLARIS.

\begin{tabular}{|c|c|c|c|}
\hline Compound & $\mathrm{Sr}_{4} \mathrm{Mn}_{2.77} \mathrm{O}_{7.16} \mathrm{Cu}_{2} \mathrm{~S}_{2}$ & $\mathrm{Sr}_{4} \mathrm{Mn}_{2.77} \mathrm{O}_{7.27} \mathrm{Cu}_{2} \mathrm{~S}_{2}$ & $\mathrm{Sr}_{4} \mathrm{Mn}_{2.77} \mathrm{O}_{7.27} \mathrm{~F}_{0.27} \mathrm{Cu}_{2} \mathrm{~S}_{2}$ \\
\hline Sample ID & GH340 & GH299 & GH336 \\
\hline Radiation & \multicolumn{3}{|c|}{ Neutron } \\
\hline Instrument & \multicolumn{3}{|c|}{ POLARIS } \\
\hline Physical form & Black powder & Black powder & Black powder \\
\hline$T / \mathrm{K}$ & 295 & 295 & 295 \\
\hline Space group & $I 4 / \mathrm{mmm}$ & $I 4 / \mathrm{mmm}$ & $I 4 / \mathrm{mmm}$ \\
\hline$a / \AA$ & $3.9012(3)$ & $3.88635(4)$ & $3.86600(5)$ \\
\hline$c / \AA$ & $34.775(3)$ & $34.5717(5)$ & $34.6478(6)$ \\
\hline$V / \AA^{3}$ & $529.3(1)$ & $522.16(2)$ & $517.84(2)$ \\
\hline $\bar{Z}$ & 2 & 2 & 2 \\
\hline$R_{w p}$ & 0.0210 & 0.0182 & 0.0229 \\
\hline$R_{F 2}$ & 0.047 & 0.053 & 0.062 \\
\hline$\chi^{2}$ & 4.997 & 2.942 & 4.809 \\
\hline
\end{tabular}


Table S7. Refined structural parameters for Mn-poor oxysulfides derived from the $\mathrm{Sr}_{4} \mathrm{Mn}_{2.77} \mathrm{O}_{7.27} \mathrm{Cu}_{2} \mathrm{~S}_{2}$ parent from PND data collected on POLARIS. $U_{\text {iso }}, U_{\text {eq }}$ measured in $\AA^{2}$

\begin{tabular}{|c|c|c|c|c|}
\hline & Compound & $\mathrm{Sr}_{4} \mathrm{Mn}_{2.77} \mathrm{O}_{7.16} \mathrm{Cu}_{2} \mathrm{~S}_{2}$ & $\mathrm{Sr}_{4} \mathrm{Mn}_{2.77} \mathrm{O}_{7.27} \mathrm{Cu}_{2} \mathrm{~S}_{2}$ & $\mathrm{Sr}_{4} \mathrm{Mn}_{2.77} \mathrm{O}_{7.27} \mathrm{~F}_{0.27} \mathrm{Cu}_{2} \mathrm{~S}_{2}$ \\
\hline & Sample ID & GH340 & GH299 & GH336 \\
\hline Atom & Parameter & & & \\
\hline \multirow[t]{2}{*}{$\operatorname{Sr} 1(1 / 21 / 2 z)$} & $z$ & $0.06354(3)$ & $0.06003(2)$ & $0.05752(3)$ \\
\hline & $100 \times U_{\mathrm{eq}}$ & $1.40(4)$ & $1.47(3)$ & $1.34(3)$ \\
\hline \multirow[t]{2}{*}{$\operatorname{Sr} 2(1 / 21 / 2 z)$} & $z$ & $0.16649(2)$ & $0.16615(2)$ & $0.16620(2)$ \\
\hline & $100 \times U_{\text {eq }}$ & $0.56(3)$ & $0.71(3)$ & $0.66(3)$ \\
\hline \multirow[t]{2}{*}{ Mn1 $\left(\begin{array}{lll}0 & 0 & z\end{array}\right)$} & $z$ & $0.11238(4)$ & $0.11238(3)$ & $0.11235(3)$ \\
\hline & $100 \times U_{\text {iso }}$ & $0.20(2)$ & $0.31(2)$ & $0.24(2)$ \\
\hline \multirow[t]{3}{*}{$\operatorname{Mn} 2(x, x, 0)$} & $x$ & $0.081(1)$ & $0^{b}$ & $0^{b}$ \\
\hline & $100 \times U_{\text {iso }}$ & $2.8(2)$ & $1.41(7)$ & $0.09(4)$ \\
\hline & Occupancy & $0.192^{b}$ & $0.769(7)$ & $0.769^{b}$ \\
\hline $\mathrm{Cu}(1 / 201 / 4)$ & $100 \times U_{\text {eq }}$ & $1.78(4)$ & $1.57(3)$ & $1.44(4)$ \\
\hline \multirow[t]{2}{*}{$\mathrm{S}\left(\begin{array}{lll}0 & 0 & z\end{array}\right)$} & $\mathrm{z}$ & $0.20792(7)$ & $0.20769(5)$ & $0.20749(6)$ \\
\hline & $100 \times U_{\text {iso }}$ & $0.74(4)$ & $0.72(3)$ & $0.79(4)$ \\
\hline
\end{tabular}




\begin{tabular}{|c|c|c|c|c|}
\hline O1 $\left(\begin{array}{lll}0 & 0 & z\end{array}\right)$ & $\mathrm{Z}$ & $0.05322(4)$ & $0.05382(3)$ & $0.05472(4)$ \\
\hline & $100 \times U_{\mathrm{eq}}$ & $2.30(5)$ & $2.42(4)$ & $1.83(4)$ \\
\hline \multirow[t]{3}{*}{$\mathrm{O} 2\left(\begin{array}{l}1 / 2 y \\
y\end{array}\right)$} & $y$ & $0.212(1)$ & $0^{b}$ & $0^{b}$ \\
\hline & $100 \times U_{\mathrm{eq}}$ & $8.6(3)$ & $8.6(2)$ & $4.0(1)$ \\
\hline & Occupancy & $0.290(4)$ & $0.636(5)$ & $0.77(6)$ \\
\hline \multirow[t]{2}{*}{$\mathrm{O} 3\left(\begin{array}{lll}1 / 2 & 0 & z\end{array}\right)$} & $z$ & $0.11967(2)$ & $0.11904(2)$ & $0.11899(2)$ \\
\hline & $100 \times U_{\text {eq }}$ & $1.05(3)$ & $0.97(2)$ & $0.87(3)$ \\
\hline
\end{tabular}

${ }^{a} U_{\text {eq }}$ is defined as one third of the orthogonalized $U_{i j}$ tensor.

${ }^{b}$ Not refined

${ }^{c}$ Total of occupancy by $\mathrm{O}$ and $\mathrm{F}$ (given the similarity in $\mathrm{O}$ and $\mathrm{F}$ scattering lengths) 
Table S8. Selected bond distances and angles for Mn-poor oxysulfides from PND data collected on POLARIS.

\begin{tabular}{|c|c|c|c|}
\hline Compound & $\mathrm{Sr}_{4} \mathrm{Mn}_{2.77} \mathrm{O}_{7.16} \mathrm{Cu}_{2} \mathrm{~S}_{2}$ & $\mathrm{Sr}_{4} \mathrm{Mn}_{2.77} \mathrm{O}_{7.27} \mathrm{Cu}_{2} \mathrm{~S}_{2}$ & $\mathrm{Sr}_{4} \mathrm{Mn}_{2.77} \mathrm{O}_{7.27} \mathrm{~F}_{0.27} \mathrm{Cu}_{2} \mathrm{~S}_{2}$ \\
\hline Sample ID & GH340 & GH299 & GH336 \\
\hline \multicolumn{4}{|l|}{ Bond distance / $\AA$} \\
\hline Mn1-O1[1] & $2.057(2)$ & $2.024(1)$ & $1.997(2)$ \\
\hline Mn1-O3 [4] & $1.9670(2)$ & $1.9568(1)$ & $1.9467(2)$ \\
\hline $\mathrm{Mn} 2-\mathrm{O} 1[2]$ & $-^{a}$ & $1.861(1)$ & $1.896(1)$ \\
\hline $\mathrm{Mn} 2-\mathrm{O} 2[4]$ & $-^{a}$ & $1.94318(2)$ & $1.93299(3)$ \\
\hline $\mathrm{Cu}-\mathrm{S}[4]$ & $2.439(1)$ & $2.4322(9)$ & $2.430(1)$ \\
\hline \multicolumn{4}{|l|}{ Bond angle $/^{\circ}$} \\
\hline O1-Mn1-O3 [4] & $97.41(4)$ & $96.76(3)$ & $96.79(4)$ \\
\hline O3-Mn1-O3 [4] & $89.05(1)$ & $89.206(7)$ & $89.199(9)$ \\
\hline $\mathrm{S}-\mathrm{Cu}-\mathrm{S}[2]$ & $106.25(9)$ & $106.06(6)$ & $105.38(8)$ \\
\hline $\mathrm{S}-\mathrm{Cu}-\mathrm{S}[4]$ & 111.11(4) & $111.21(3)$ & $111.55(4)$ \\
\hline
\end{tabular}

${ }^{a}$ omitted due to structural disorder in $\mathrm{O} 2$ and $\mathrm{Mn} 2$ positions 
Table S9. Results of PND refinements at $295 \mathrm{~K}$ for oxyselenides from PND data collected on POLARIS.

\begin{tabular}{|c|c|c|c|c|}
\hline Compound & $\mathrm{Sr}_{4} \mathrm{Mn}_{3} \mathrm{O}_{7.35} \mathrm{Cu}_{2} \mathrm{Se}_{2}$ & $\mathrm{Sr}_{4} \mathrm{Mn}_{3} \mathrm{O}_{7.56} \mathrm{Cu}_{2} \mathrm{Se}_{2}$ & $\mathrm{Sr}_{4} \mathrm{Mn}_{3} \mathrm{O}_{7.53} \mathrm{Cu}_{2} \mathrm{Se}_{2}$ & $\mathrm{Sr}_{4} \mathrm{Mn}_{3} \mathrm{O}_{7.53} \mathrm{~F}_{0.20} \mathrm{Cu}_{2} \mathrm{Se}_{2}$ \\
\hline Sample ID & GH198 & GH201 & GH288 & GH319 \\
\hline Radiation & \multicolumn{4}{|c|}{ Neutron } \\
\hline Instrument & \multicolumn{4}{|c|}{ POLARIS } \\
\hline Physical form & Black powder & Black powder & Black powder & Black powder \\
\hline$T / \mathrm{K}$ & 295 & 295 & 295 & 295 \\
\hline Space group & $I 4 / \mathrm{mmm}$ & $I 4 / \mathrm{mmm}$ & $I 4 / \mathrm{mmm}$ & $I 4 / \mathrm{mmm}$ \\
\hline$a / \AA$ & $3.917(1)$ & $3.900(1)$ & $3.89217(4)$ & $3.8848(8)$ \\
\hline$c / \AA$ & $35.33(1)$ & $35.44(1)$ & $35.3852(4)$ & $35.414(7)$ \\
\hline$V / \AA^{3}$ & $541.9(5)$ & $539.1(5)$ & $536.05(2)$ & $534.5(3)$ \\
\hline $\mathrm{Z}$ & 2 & 2 & 2 & 2 \\
\hline$R_{w p}$ & 0.0197 & 0.0259 & 0.0185 & 0.0240 \\
\hline$R_{F 2}$ & 0.075 & 0.055 & 0.044 & 0.054 \\
\hline$\chi^{2}$ & 4.558 & 4.646 & 3.296 & 5.017 \\
\hline
\end{tabular}


Table S10. Refined structural parameters for oxyselenides from PND data collected on POLARIS. $U_{\text {iso }}, U_{\text {eq }}$ measured in $\AA^{2}$.

\begin{tabular}{|c|c|c|c|c|c|}
\hline & Compound & $\mathrm{Sr}_{4} \mathrm{Mn}_{3} \mathrm{O}_{7.35} \mathrm{Cu}_{2} \mathrm{Se}_{2}$ & $\mathrm{Sr}_{4} \mathrm{Mn}_{3} \mathrm{O}_{7.56} \mathrm{Cu}_{2} \mathrm{Se}_{2}$ & $\mathrm{Sr}_{4} \mathrm{Mn}_{3} \mathrm{O}_{7.53} \mathrm{Cu}_{2} \mathrm{Se}_{2}$ & $\mathrm{Sr}_{4} \mathrm{Mn}_{3} \mathrm{O}_{7.53} \mathrm{~F}_{0.20} \mathrm{Cu}_{2} \mathrm{Se}_{2}$ \\
\hline & Sample ID & GH198 & GH201 & GH288 & GH319 \\
\hline Atom & Parameter & & & & \\
\hline \multirow[t]{2}{*}{$\operatorname{Sr} 1(1 / 21 / 2 z)$} & $z$ & $0.05804(4)$ & $0.05645(2)$ & $0.05635(2)$ & $0.05496(3)$ \\
\hline & $100 \times U_{\mathrm{eq}}$ & $1.81(6)$ & $1.29(3)$ & $1.16(3)$ & $1.24(3)$ \\
\hline \multirow[t]{2}{*}{$\operatorname{Sr} 2(1 / 21 / 2 z)$} & $z$ & $0.16232(3)$ & $0.16209(2)$ & $0.16204(2)$ & $0.16211(2)$ \\
\hline & $100 \times U_{\text {eq }}$ & $0.51(2)$ & $0.71(2)$ & $0.68(2)$ & $0.62(3)$ \\
\hline \multirow[t]{2}{*}{ Mn1 $\left(\begin{array}{lllllll}0 & 0 & z\end{array}\right)$} & $z$ & $0.10900(5)$ & $0.10940(3)$ & $0.10924(3)$ & $0.10908(4)$ \\
\hline & $100 \times U_{\text {iso }}$ & $0.45(3)$ & $0.41(2)$ & $0.41(2)$ & $0.42(2)$ \\
\hline $\operatorname{Mn} 2(0,0,0)$ & $100 \times U_{\text {iso }}$ & $1.72(7)$ & $0.74(3)$ & $0.62(3)$ & $0.60(4)$ \\
\hline $\mathrm{Cu}(1 / 201 / 4)$ & $100 \times U_{\text {eq }}$ & $1.52(5)$ & $1.41(2)$ & $1.34(2)$ & $1.49(3)$ \\
\hline \multirow[t]{2}{*}{$\operatorname{Se}\left(\begin{array}{lll}0 & 0 & z\end{array}\right)$} & $\mathrm{z}$ & $0.20591(3)$ & $0.20555(2)$ & $0.20549(2)$ & $0.20535(2)$ \\
\hline & $100 \times U_{\text {eq }}$ & $0.98(3)$ & $0.85(2)$ & $0.74(2)$ & $0.77(3)$ \\
\hline \multirow[t]{2}{*}{ O1 $\left(\begin{array}{llllll}0 & 0 & z\end{array}\right)$} & $\mathrm{z}$ & $0.05205(5)$ & $0.05305(3)$ & $0.05301(3)$ & $0.05293(4)$ \\
\hline & $100 \times U_{\text {eq }}$ & $2.44(8)$ & $2.17(4)$ & $2.08(4)$ & $1.47(4)$ \\
\hline $\mathrm{O} 2\left(\begin{array}{llll}1 / 2 & 0 & 0\end{array}\right)$ & $100 \times U_{\text {eq }}$ & $8.7(3)$ & $4.2(1)$ & $3.7(1)$ & $2.0(1)$ \\
\hline
\end{tabular}




\begin{tabular}{|l|l|l|l|l|l|}
\hline & Occupancy & $0.67(1)$ & $0.779(5)$ & $0.766(4)$ & $0.864(5)^{c}$ \\
\hline O3 (1/2 0 z) & $z$ & $0.11592(3)$ & $0.11613(2)$ & $0.11613(1)$ & $0.11599(2)$ \\
\hline & $U_{\mathrm{eq}}$ & $1.14(5)$ & $1.08(2)$ & $0.97(2)$ & $0.87(3)$ \\
\hline
\end{tabular}

${ }^{a} U_{\text {eq }}$ is defined as one third of the orthogonalized $U_{i j}$ tensor.

${ }^{b}$ Not refined

${ }^{c}$ Total of occupancy by $\mathrm{O}$ and $\mathrm{F}$ (given the similarity in $\mathrm{O}$ and $\mathrm{F}$ scattering lengths) 
Table S11. Selected bond distances and angles for oxyselenides from PND data collected on POLARIS.

\begin{tabular}{|c|c|c|c|c|}
\hline Compound & $\mathrm{Sr}_{4} \mathrm{Mn}_{3} \mathrm{O}_{7.35} \mathrm{Cu}_{2} \mathrm{Se}_{2}$ & $\mathrm{Sr}_{4} \mathrm{Mn}_{3} \mathrm{O}_{7.56} \mathrm{Cu}_{2} \mathrm{Se}_{2}$ & $\mathrm{Sr}_{4} \mathrm{Mn}_{3} \mathrm{O}_{7.53} \mathrm{Cu}_{2} \mathrm{Se}_{2}$ & $\mathrm{Sr}_{4} \mathrm{Mn}_{3} \mathrm{O}_{7.53} \mathrm{~F}_{0.20} \mathrm{Cu}_{2} \mathrm{Se}_{2}$ \\
\hline Sample ID & GH198 & GH201 & GH288 & GH319 \\
\hline \multicolumn{5}{|l|}{ Bond distance / $\AA$} \\
\hline Mn1-O1[1] & $2.012(3)$ & $1.997(2)$ & $1.990(1)$ & $1.989(2)$ \\
\hline Mn1-O3 [4] & $1.9736(7)$ & $1.965(6)$ & $1.9613(1)$ & $1.9577(4)$ \\
\hline $\mathrm{Mn} 2-\mathrm{O} 1[2]$ & $1.839(2)$ & $1.880(1)$ & $1.876(1)$ & $1.874(1)$ \\
\hline $\mathrm{Mn} 2-\mathrm{O} 2[4]$ & $1.9584(6)$ & $1.9502(6)$ & $1.94608(2)$ & $1.9424(4)$ \\
\hline $\mathrm{Cu}-\mathrm{Se}[4]$ & $2.5023(9)$ & $2.5069(7)$ & $2.5037(4)$ & $2.5046(6)$ \\
\hline \multicolumn{5}{|l|}{ Bond angle $/{ }^{\circ}$} \\
\hline O1-Mn1-O3 [4] & $97.11(6)$ & $96.98(3)$ & $97.14(3)$ & $97.18(4)$ \\
\hline $\mathrm{O} 3-\mathrm{Mn} 1-\mathrm{O} 3$ [4] & $89.12(1)$ & $89.155(8)$ & $89.115(7)$ & $89.11(1)$ \\
\hline $\mathrm{Se}-\mathrm{Cu}-\mathrm{Se}[2]$ & $103.01(5)$ & $102.14(3)$ & $102.03(2)$ & 101.71(1) \\
\hline $\mathrm{Se}-\mathrm{Cu}-\mathrm{Se}[4]$ & $112.80(3)$ & $113.26(2)$ & $113.32(1)$ & $113.49(2)$ \\
\hline
\end{tabular}


Figure S1. Electrical resitivity of a sample of $\mathrm{Sr}_{4} \mathrm{Mn}_{2.9} \mathrm{O}_{7.4} \mathrm{Cu}_{2} \mathrm{~S}_{2}$ as a function of temperature measured using a 4-probe technique.

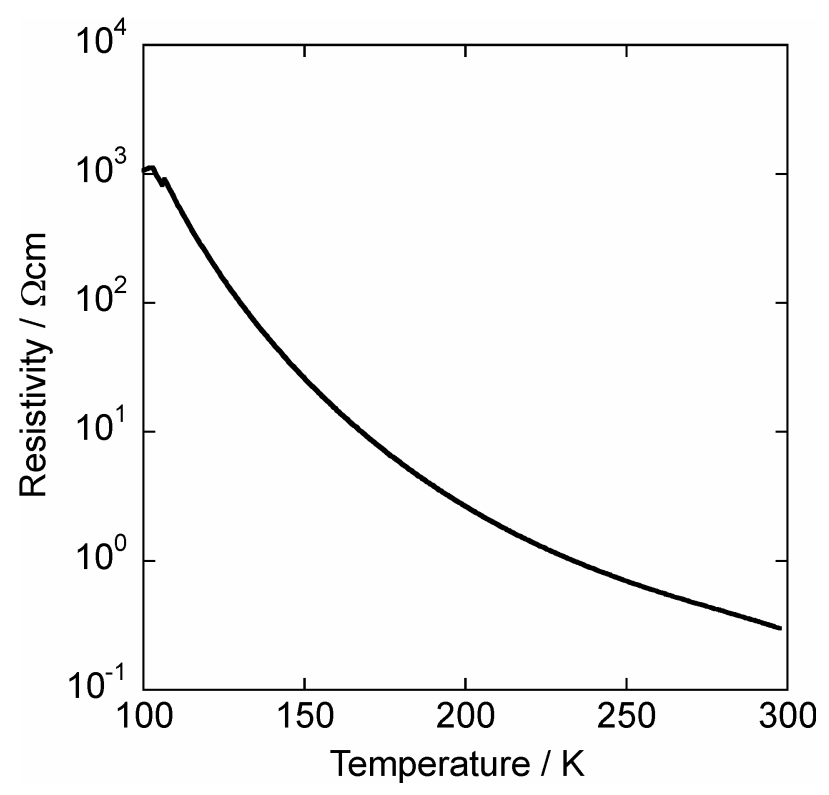


Figure S2. The crystal structure of $\mathrm{Sr}_{4} \mathrm{Mn}_{2.94(2)} \mathrm{O}_{7.42(5)} \mathrm{Cu}_{2} \mathrm{~S}_{2}$ derived from single crystal X-ray diffraction data.

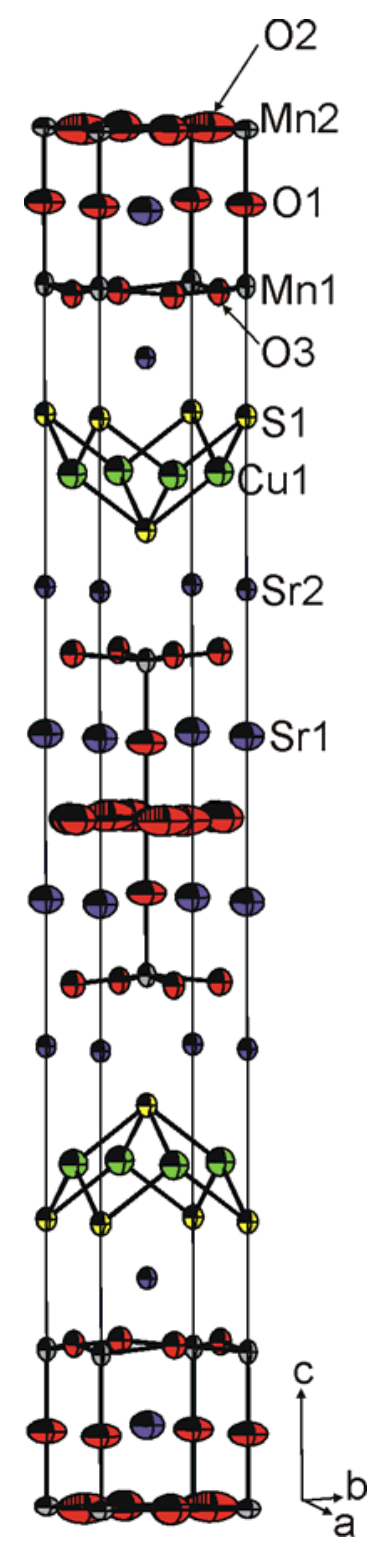


Figure S3. Electron diffraction patterns for the as-made Mn-rich oxysulfide $\mathrm{Sr}_{4} \mathrm{Mn}_{2.91} \mathrm{O}_{7.40} \mathrm{Cu}_{2} \mathrm{~S}_{2}$ showing no signs of vacancy/anion ordering leading to a superstructure on the lengthscale of the electron diffraction experiment. The patterns are indexed on a body centred tetragonal unit cell with the dimensions given in Table 1.
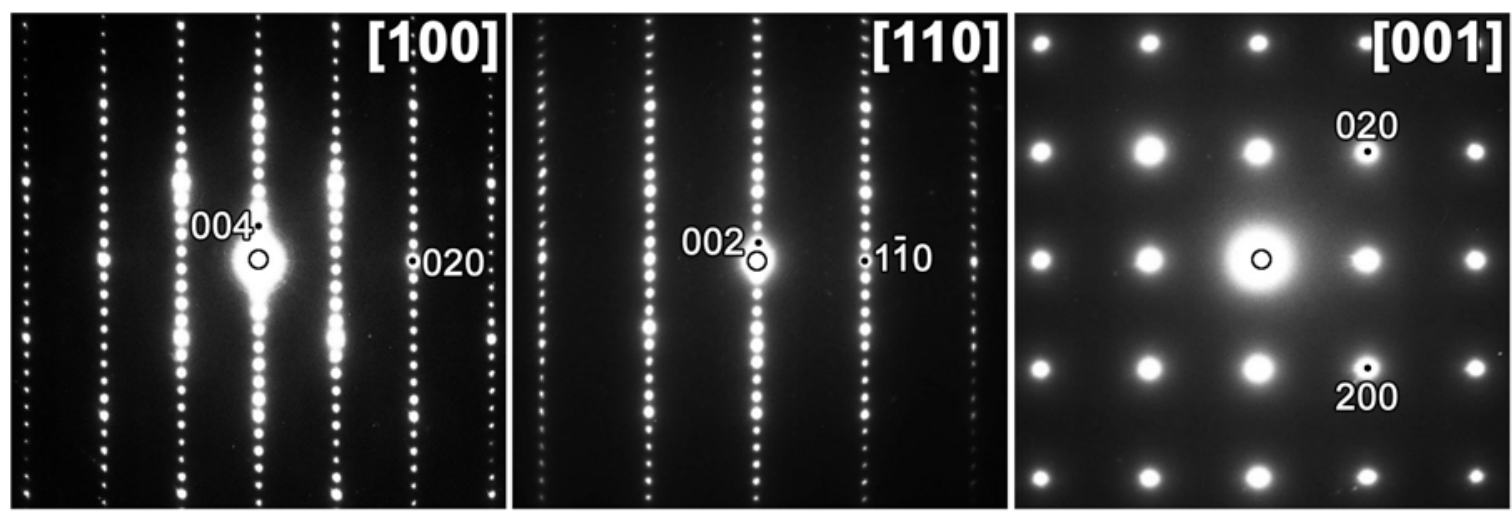
Figure S4. Comparative thermogravimetric analysis results for $\mathrm{Sr}_{4} \mathrm{Mn}_{2.91} \mathrm{O}_{7.40} \mathrm{Cu}_{2} \mathrm{~S}_{2}$ and its reduced derivative $\mathrm{Sr}_{4} \mathrm{Mn}_{2.91} \mathrm{O}_{6.97} \mathrm{Cu}_{2} \mathrm{~S}_{2}$.

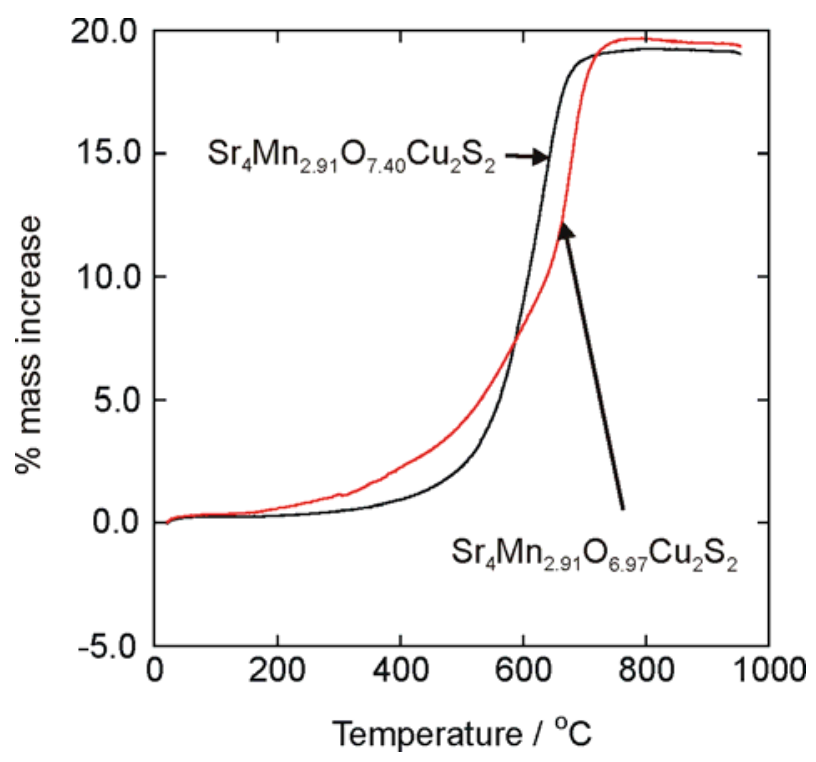


Figure S5. The environments around $\mathrm{Mn} 2$ in the central layer obtained by Rietveld refinement against Powder Neutron Diffraction Data collected on the POLARIS diffractometer. As-made, reduced and fluorinated Mn-rich oxysulfides and oxyselenides are shown as a function of the refined fractional occupancy of the $\mathrm{O} 2$ site. Displacement ellipsoids are shown at the $80 \%$ level

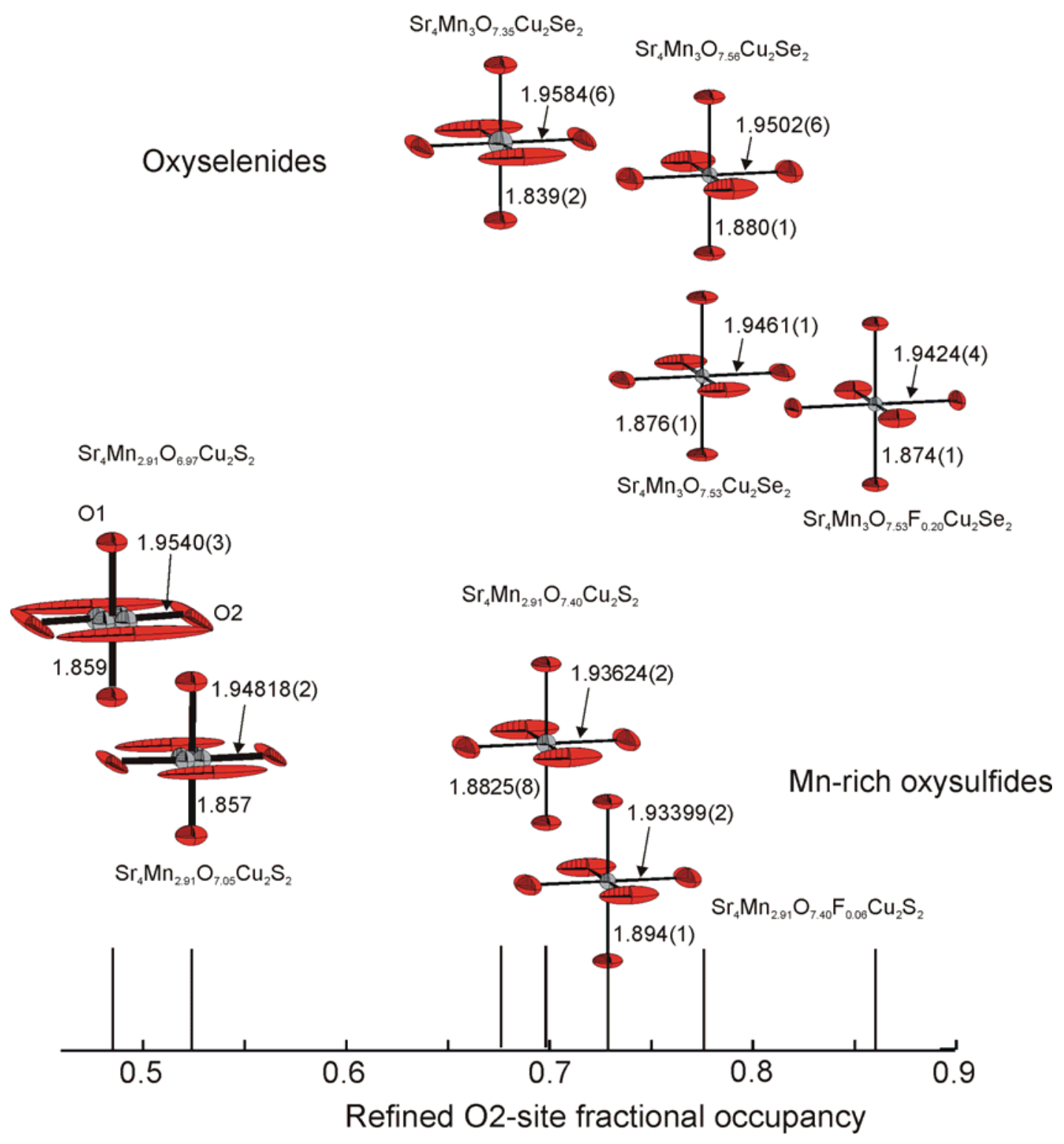


Figure S6. The magnetic susceptibilities of Mn-poor oxysulfides: as-made $\mathrm{Sr}_{4} \mathrm{Mn}_{2.77} \mathrm{O}_{7.27} \mathrm{Cu}_{2} \mathrm{~S}_{2}$ (black), reduced $\mathrm{Sr}_{4} \mathrm{Mn}_{2.77} \mathrm{O}_{7.16} \mathrm{Cu}_{2} \mathrm{~S}_{2}$ (green) and oxidized $\mathrm{Sr}_{4} \mathrm{Mn}_{2.77} \mathrm{O}_{7.27} \mathrm{~F}_{0.27} \mathrm{Cu}_{2} \mathrm{~S}_{2}$ (blue). Samples were measured under Zero-Field-Cooled (ZFC) (closed symbols) and Field-Cooled (FC) (open symbols) conditions in a measuring field of $0.01 \mathrm{~T}$. The discernable maximum equated with the Neél temperatures for $\mathrm{Sr}_{4} \mathrm{Mn}_{2.77} \mathrm{O}_{7.27} \mathrm{~F}_{0.27} \mathrm{Cu}_{2} \mathrm{~S}_{2}$ is indicated by an arrow.

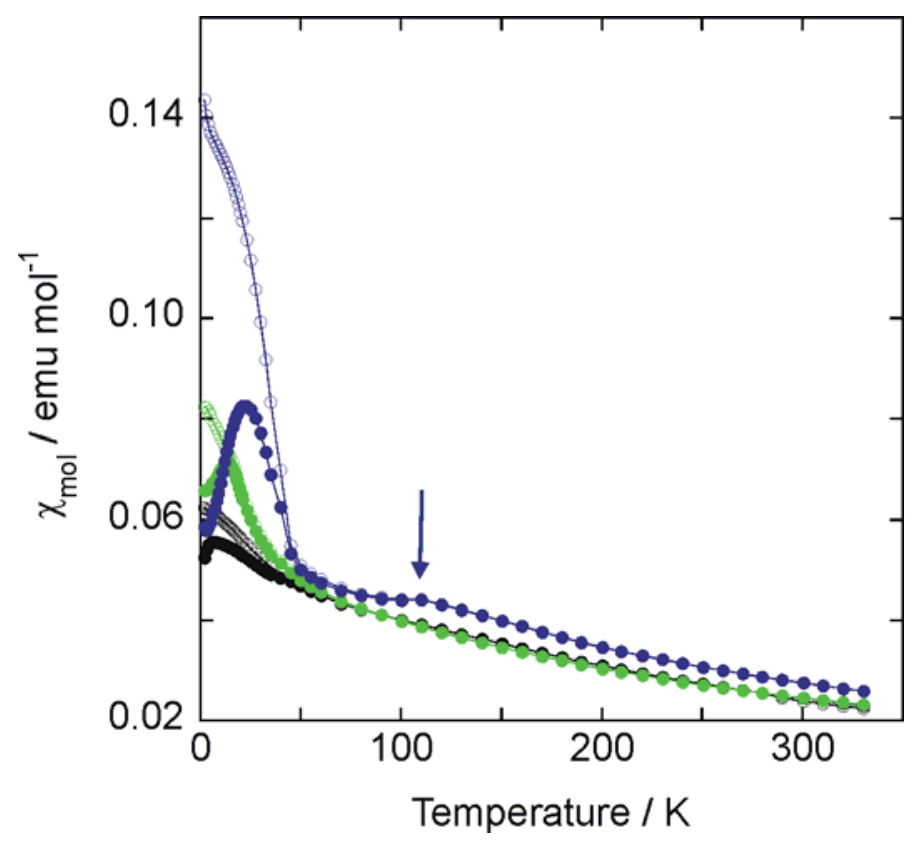


Figure S7. Upper: 5K Magnetization isotherms for Mn-poor oxysulfides: As-made $\mathrm{Sr}_{4} \mathrm{Mn}_{2.77} \mathrm{O}_{7.27} \mathrm{Cu}_{2} \mathrm{~S}_{2}$ (black), its reduction product $\mathrm{Sr}_{4} \mathrm{Mn}_{2.77} \mathrm{O}_{7.16} \mathrm{Cu}_{2} \mathrm{~S}_{2}$ (green) and its oxidation product $\mathrm{Sr}_{4} \mathrm{Mn}_{2.77} \mathrm{O}_{7.27} \mathrm{~F}_{0.27} \mathrm{Cu}_{2} \mathrm{~S}_{2}$ (blue). Lower: $5 \mathrm{~K}$ magnetization isotherms for oxyselenides: as-made $\mathrm{Sr}_{4} \mathrm{Mn}_{3} \mathrm{O}_{7.56} \mathrm{Cu}_{2} \mathrm{Se}_{2}$ (black, closed circles) and its reduction product $\mathrm{Sr}_{4} \mathrm{Mn}_{3} \mathrm{O}_{7.35} \mathrm{Cu}_{2} \mathrm{Se}_{2}$ (green closed circles); and as-made $\mathrm{Sr}_{4} \mathrm{Mn}_{3} \mathrm{O}_{7.53} \mathrm{Cu}_{2} \mathrm{Se}_{2}$ (black open circles) and its oxidation product $\mathrm{Sr}_{4} \mathrm{Mn}_{3} \mathrm{O}_{7.53} \mathrm{~F}_{0.20} \mathrm{Cu}_{2} \mathrm{Se}_{2}$ (blue open circles).
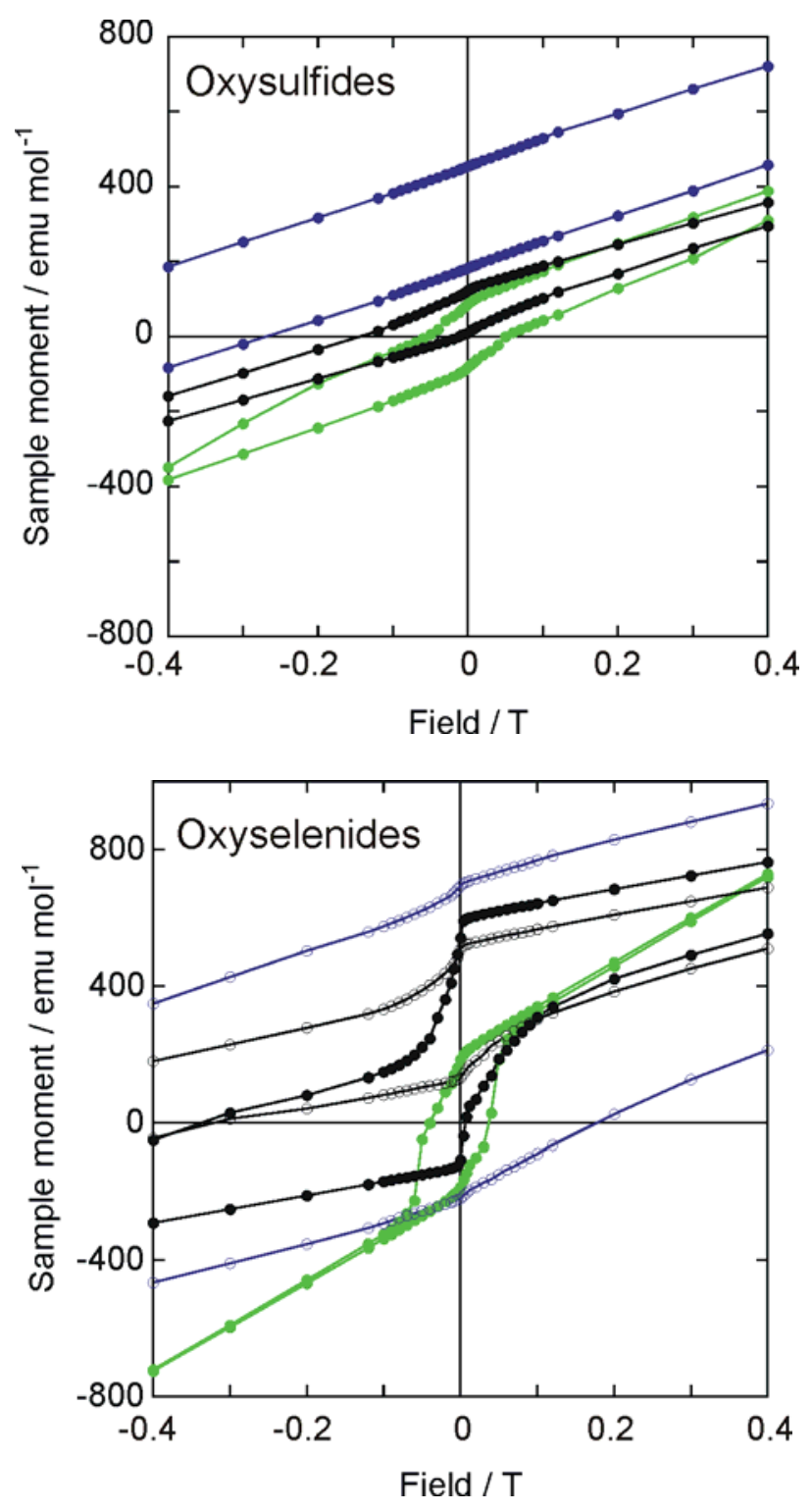